\title{
A Novel Method for the On-Line Room-Temperature Hydrolysis of Hydrophobic Organic Phosphates Using a Cooperative Action of Sodium Dodecyl Sulfate, Acid and UV-Irradiation
}

\author{
Yoshihiko IKEgUCHI and Hiroshi NAKAMURA ${ }^{\dagger}$ \\ Department of Analytical Chemistry, Faculty of Pharmaceutical Sciences, Science University of Tokyo, \\ Ichigaya-funagawara-machi, Shinjuku, Tokyo 162-0826, Japan
}

Keywords Hydrophobic organic phosphate, sodium dodecyl sulfate, on-line hydrolysis, room-temperature hydrolysis, UV-irradiation, flow injection analysis, phosphomolybdic acid reaction, thiamine-thiochrome reaction

Recently, the determination of various organic phosphates in environmental and biological samples has been urgently desired due to their importance in environmental and biomedical fields. Organic phosphates are usually determined by a phosphomolybdic acid reaction after conversion to orthophosphates. Some methods have been reported for the on-line decomposition of organic phosphates using cobalt(III) oxidation ${ }^{1}$, peroxodisulfate digestion ${ }^{2,3}$, enzymatic dephosphorylation $^{4}$, and oxidative decomposition with light ${ }^{5,6}$ and microwaves. ${ }^{7,8}$ We also reported on the on-line hydrolysis of organic phosphates by sulfuric acid ${ }^{9}$ and its application $^{10-12}$ to their determination. The organic phosphates examined so far were all water-soluble. Although Bessman and his co-workers ${ }^{13-15}$ reported on the automated determination of organic phosphates, including some hydrophobic compounds, like phospholipids, by a Molybdenum-Blue method after ashing organic phosphates, there is no general method for the on-line determination of hydrophobic organic phosphates, except for liquid chromatography with mass spectrometry ${ }^{16}$ and flame photometric detection. ${ }^{17}$ In this communication, we therefore describe the finding that some hydrophobic organic phosphates are easily decomposed in an acidic medium containing sodium dodecyl sulfate, under UV-irradiation, without heating or any oxidizing agent added in solutions. The new decomposition system was evaluated by measuring orthophosphate liberated from organic phosphates in a flow injection system, in which a phosphomolybdic acid reaction and then a thiamine-thiochrome fluorescent reaction ${ }^{18}$ occurred.

\section{Experimental}

\section{Materials and reagents}

All of the reagents purchased were of guaranteed grade and were used without further purification.

\footnotetext{
$\doteqdot$ To whom correspondence should be addressed.
}

Organic phosphates were dissolved in water containing $0.5 \%(\mathrm{w} / \mathrm{v})$ sodium lauryl sulfate (SDS) to make 100 $\mu \mathrm{M}$ sample solutions, except for $0.1 \mathrm{mg} \mathrm{m}^{-1}$ for lysophosphatidyl-ethanolamine (LPE) (pig liver).

\section{FIA system}

Figure 1 shows the FIA system used in this experiment for the determination of hydrophobic organic phosphates. A carrier (A) containing $0.5 \%(\mathrm{w} / \mathrm{v})$ SDS was delivered at a flow rate of $0.5 \mathrm{ml} \mathrm{min} \mathrm{m}^{-1}$ with a JASCO 880-PU pump (Tokyo, Japan) $\left(\mathrm{P}_{1}\right)$. An acidic solution (B), a molybdenum reagent solution (C) and a thiamine (D) in an alkaline solution (E) were pumped with a Shimadzu LC-9A pump (Kyoto, Japan) $\left(\mathrm{P}_{2}\right)$ at

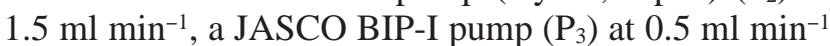

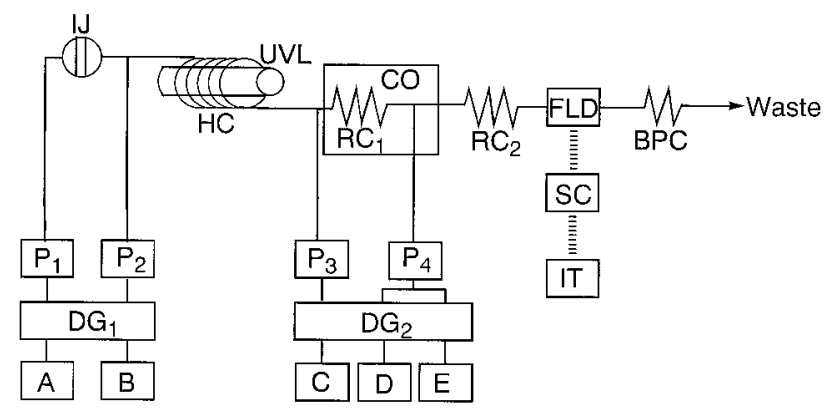

Fig. 1 Schematic diagram of the FIA system for hydrophobic organic phosphates. A, $0.5 \%(\mathrm{w} / \mathrm{v}) \mathrm{SDS} ; \mathrm{B}, 0.2 \%(\mathrm{v} / \mathrm{v})$ trifluoroacetic acid (TFA); $\mathrm{C}, 0.75 \mathrm{M} \mathrm{H}_{2} \mathrm{SO}_{4}$ and $2.5 \%(\mathrm{w} / \mathrm{v})$ ammonium molybdate; $\mathrm{D}, 1.2 \%(\mathrm{w} / \mathrm{v})$ thiamine hydrochloride (thiamine); E, 5.3 M NaOH and $25 \mathrm{mM} \mathrm{Na}_{2} \mathrm{~B}_{4} \mathrm{O}_{7} . \mathrm{DG}_{1}$, degassar (DG-980-50, JASCO, Tokyo, Japan); $\mathrm{P}_{1}$, pump (0.5 $\left.\mathrm{ml} \mathrm{min}^{-1}\right)$; IJ, injector; $\mathrm{P}_{2}$, pump $\left(1.5 \mathrm{ml} \mathrm{min}^{-1}\right)$; UVL, $20 \mathrm{~W}$ UV lamp; $\mathrm{HC}$, hydrolysis coil; $\mathrm{DG}_{2}$, degassar (ERC-3512, Erma, Tokyo, Japan); $\mathrm{P}_{3}$, pump (0.5 $\left.\mathrm{ml} \mathrm{min}^{-1}\right)$; $\mathrm{CO}$, column oven; $\mathrm{RC}_{1}$,

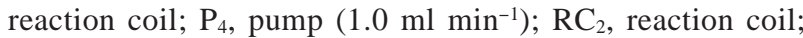
FLD, fluorescence detector (excitation wavelength $375 \mathrm{~nm}$; emission wavelength $440 \mathrm{~nm}$ ); SC, signal cleaner (SC77, Tomsic, Tokyo, Japan); IT, integrator (807-IT, JASCO); BPC, back-pressure coil $(0.25 \mathrm{~mm}$ i.d. $\times 0.75 \mathrm{~m})$. 
and a Shimadzu LC-9A pump $\left(\mathrm{P}_{4}\right)$ with a FCV-9AL low-pressure gradient-elution flow-control valve $(\mathrm{D} / \mathrm{E}=50 / 50)$ (Shimadzu) at $1.0 \mathrm{ml} \mathrm{min}{ }^{-1}$, respectively. A $30 \mathrm{~m}$-hydrolysis coil (PTFE; white color in appearance; commonly available in the market; inner diameter, $0.5 \mathrm{~mm}$; outer diameter, $1.5 \mathrm{~mm}$; pretreated by delivering a $1.0 \%(\mathrm{w} / \mathrm{v}) \mathrm{SDS}$ solution of $30 \mathrm{mg} / \mathrm{l} \mathrm{TiO}$ particles (below $5 \mu \mathrm{m}$ anatase form; Wako, Osaka, Japan)) (HC) was wound around a UV-lamp (20 W sterilizing lamp; bright line wavelength, $253.7 \mathrm{~nm}$; Toshiba GL20; Tokyo, Japan) (UVL). Ten microliters of the sample solution were introduced into the carrier stream via a Rheodyne 7125 injector (Cotati, CA, USA) (IJ) with a $10 \mu \mathrm{l}$-microsyringe. Organic phosphates introduced into the hydrolysis coil were irradiated by the UV-lamp in order to liberate orthophosphate. The resultant orthophosphate was reacted in a reaction coil $(0.5 \mathrm{~mm}$ i.d. $\times 1.5 \mathrm{~m})\left(\mathrm{RC}_{1}\right)$ at $30^{\circ} \mathrm{C}$ in a column oven (CTO-2A, Shimadzu) (CO) with a molybdenum reagent to form the phosphomolybdic acid, which was then reacted in a reaction coil $(0.5 \mathrm{~mm}$ i.d. $\times 1.0 \mathrm{~m})$ $\left(\mathrm{RC}_{2}\right)$ with the alkaline thiamine reagent at room temperature. Finally, the fluorescence intensity of the thiochrome converted from thiamine by phosphomolybdic acid was measured at the excitation wavelength $(375 \mathrm{~nm})$ and emission wavelength $(440 \mathrm{~nm})$ with a JASCO FP920 fluorescence detector (FLD). The series of reactions employed in our detection system is illustrated in Scheme 1. The FIA system, using $0.2 \%(\mathrm{v} / \mathrm{v})$ TFA as the B-solvent, gave good linearity (correlation coefficient $\approx 0.998$ ) from 10 to $250 \mu \mathrm{M}$ (injected volume, $10 \mu \mathrm{l}$ ) of orthophosphate and enabled the analysis of 20 samples $\mathrm{h}^{-1}$ with a relative standard deviation of $2.36 \%(n=10)$. The detection limit $(S / N=3)$ of orthophosphate was 50 pmol.

The relative molar response of a $100 \mu \mathrm{M}$ sample solution, except for $0.1 \mathrm{mg} \mathrm{ml}^{-1} \mathrm{LPE}$ dissolved in a $0.5 \%$ SDS solution was calculated by comparing the peak area with a calibration curve of an authentic orthophosphate solution prepared with $0.5 \%$ SDS, taking the relative molar response of $100 \mu \mathrm{M}$ orthophosphate as 100 .

\section{Results and Discussion}

As shown in Fig. 2, 0.2\% was optimal as the TFA concentration in the B-solvent, by which LPE and didecyl-P were mostly decomposed under UV light after mixing with $0.5 \%$ SDS in the A-solvent. When either SDS, TFA or UV-irradiation was deleted from the FIA system, no organic phosphate could be detected. The concentration of SDS in the A-solvent as a carrier was optimal at $0.5 \%$; neither lower or higher concentrations were effective for the decomposition. Since Table 1 illustrates the effect of some acids on the decomposition of organic phosphates, and Fig. 2 indicates that the hydrolysis efficiency is effected by the TFA concentration, the decomposition was considered to depend not only on the kind of acids, but also on the acidity, itself, more or less. While $O$-phospho-L-threonine, D-ribose5-phosphate and $O$-phosphoryl-ethanolamine, tested as model compounds of hydrophilic organic phosphates, were not detectable, LPE, dodecyl-P, di-decyl-P and PC

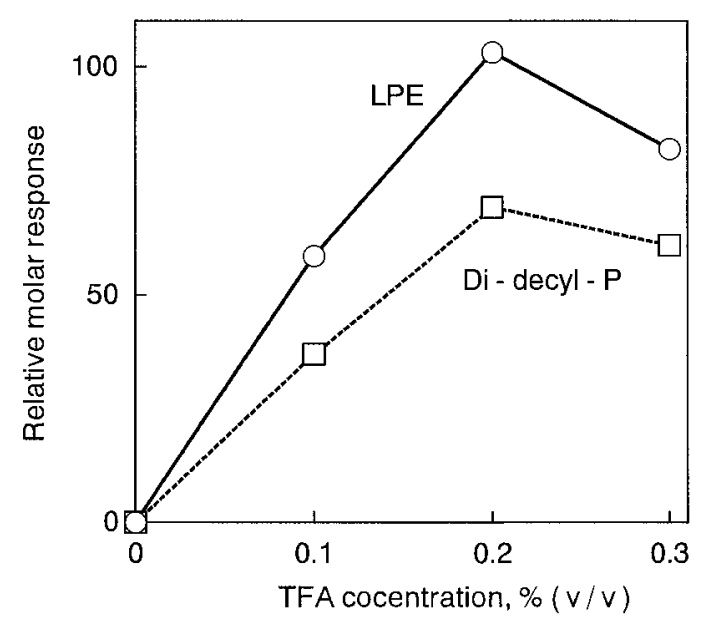

Fig. 2 Effect of the TFA concentration on the decomposition of hydrophobic organic phosphates under UV-irradiation. Asolvent, $0.5 \%(\mathrm{w} / \mathrm{v}) \mathrm{SDS}$.

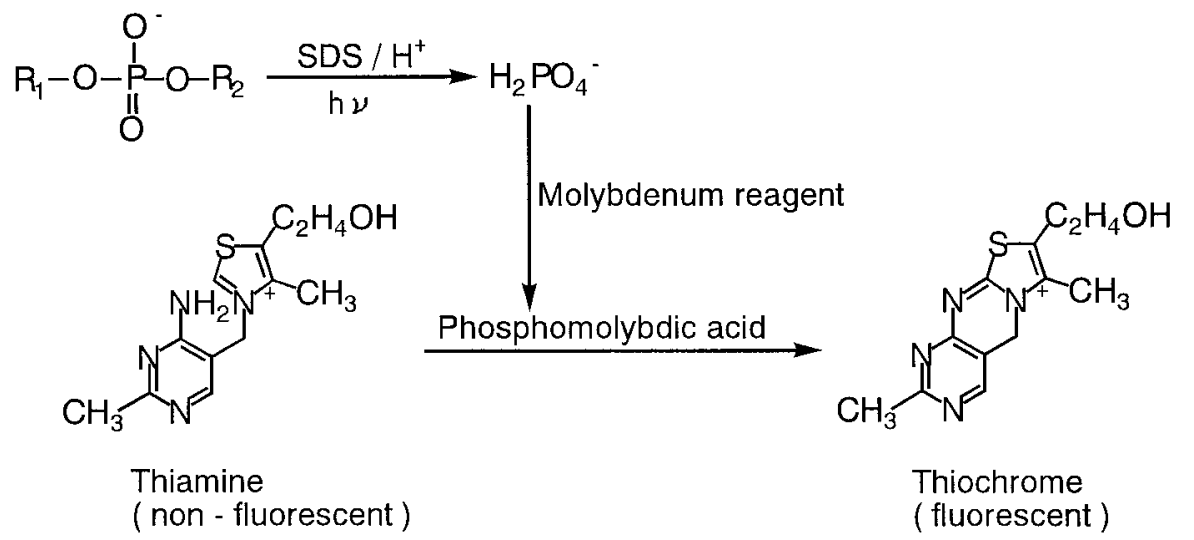

Scheme 1 Reaction scheme for the determination of hydrophobic organic phosphates. 
Table 1 Effect of acids on decomposition of hydrophobic organic phosphates under UV-irradiation ${ }^{\mathrm{a}}$

\begin{tabular}{lccc}
\hline \multirow{3}{*}{ Compound } & \multicolumn{3}{c}{ Relative molar response } \\
\cline { 2 - 4 } & $0.2 \%$ TFA & $10 \mathrm{mM} \mathrm{H}_{2} \mathrm{SO}_{4}$ & $0.1 \%$ Acetic acid \\
\hline LPE & 103.2 & 94.7 & 71.0 \\
Dodecyl-P & 87.3 & 90.1 & 65.6 \\
Di-decyl-P & 69.3 & 74.5 & 63.4 \\
PC & 37.4 & 40.3 & 35.1 \\
\hline
\end{tabular}

a. After mixing with A-solvent containing $0.5 \%(\mathrm{w} / \mathrm{v}) \mathrm{SDS}$, the decomposition was performed using a 30-m coil. Dodecyl-P, phosphoric acid mono- $n$-dodecyl ester; Di-decyl-P, phosphoric acid di- $n$-decylester PC, L- $\alpha$-phosphatidylcholine dipalmitoyl.

as model compounds of hydrophobic ones were successfully detected. This result indicates that the decomposition assisted by UV-irradiation, SDS and TFA was limited to the hydrophobic organic phosphates, even though the decomposition was not completed.

The FIA system gave good linearity (correlation coefficient $\approx 0.997$ ) from 0.01 to $0.5 \mathrm{mg} \mathrm{ml}^{-1}$ (injected volume, $10 \mu \mathrm{l})$ of LPE and enabled the analysis of $15 \mathrm{sam}-$ ples $\mathrm{h}^{-1}$ with a relative standard deviation of $3.53 \%$ $(n=10)$. The detection limit of LPE was $0.05 \mu \mathrm{g}$ $(S / N=3)$.

In conclusion, the present method using both UV-irradiation and a mixture of SDS and acid, without heating or any oxidizing agent added in solutions, is quite unique. Since there is no practical on-line hydrolysis method for hydrophobic organic phosphates, the present method will be successfully applicable for various purposes.

\section{References}

1. H. Tanaka, T. Fukuoka and K. Okamoto, Anal. Sci., 11, 787 (1995)

2. T. Korenaga and K. Okada, Bunseki Kagaku, 33, 683 (1984).

3. M. Goto, M. Nishimura, T. Tajima and D. Ishii, Bunseki Kagaku, 37, 52 (1988).

4. T. Fujiwara, K. Kurahashi and T. Kumamaru, Anal. Chim. Acta, 349, 159 (1997)

5. K. Hiiro, A. Kawahara, T. Tanaka and S. Wakida, Bunseki Kagaku, 33, 510 (1984)

6. I. D. McKelvie, B. T. Hart, T. J. Cardwell and R. W. Cattrall, Analyst[London], 114, 1459 (1989).

7. K. E. Williams, S. J. Haswell, D. A. Barclay and G. Preston, Analyst [London], 118, 245 (1993).

8. S. Hinkamp and G. Schwedt, Anal. Chim. Acta, 236, 345 (1990).

9. Y. Ikeguchi, H. Nakamura and T. Nakajima, Anal. Sci., 9, 397 (1993).

10. Y. Ikeguchi, H. Nakamura and T. Nakajima, Anal. Sci., 9, 653 (1993).

11. Y. Ikeguchi and H. Nakamura, Anal. Sci., 13, 479 (1997).

12. S. Takahashi and H.Nakamura, Anal. Sci., 13, 601 (1997).

13. S. P. Bessman, Anal. Biochem., 59, 524 (1974).

14. J. K. Kaitaranta, P. J. Geiger and S. P. Bessman, J. Chromatogr., 206, 327 (1981).

15. J. K. Kaitaranta and S. P. Bessman, Anal. Chem., 53, 1232 (1981).

16. H.-Y. Kim, T.-C. L. Wang and Y.-C. Ma, Anal. Chem., 66, 3977 (1994).

17. T. L. Chester, Anal. Chem., 52, 1621 (1980).

18. P. Linares, M. D. L. de Castro and M. Valcarcel, Talanta, 33, 889 (1986)

(Received March 11, 1998) (Accepted June 8, 1998) 